\title{
The Gown-glove Interface Is a Source of Contamination: A Comparative Study
}

\author{
James F. Fraser MD, MPH, Simon W. Young FRACS, \\ Kimberly A. Valentine RN, Nicholas E. Probst PA-C, \\ Mark J. Spangehl MD
}

Published online: 9 December 2014

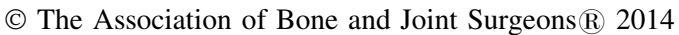

\begin{abstract}
Background The original Charnley-type negative-pressure body exhaust suit reduced infection rates in randomized trials of total joint arthroplasty (TJA) decades ago. However, modern positive-pressure surgical helmet systems have not shown similar benefit, and several recent studies have raised the question of whether these gowning systems result in increased wound contamination and infections. The gown-glove interface may be one source of particle contamination.
\end{abstract}

One of the authors certifies that he (JFF), or a member of his family, has or may receive payments or benefits, during the study period, of an amount of USD 10,000 to USD 100,000 from Stryker (Kalamazoo, MI, USA). One of the authors (SWY) certifies that he, or a member of his immediate family, has or may receive payments or benefits, during the study period, of an amount of USD 10,000 to USD 100,000 from Vidacare (San Antonio, TX, USA). One of the authors (MJS) certifies that he, or a member of his immediate family, has or may receive payments or benefits, during the study period, of an amount of USD 10,000 to USD 100,000 from Stryker, USD 10,000 to USD 100,000 from DePuy Synthes (West Chester, PA, USA), and USD 10,000 to USD 100,000 from Vidacare.

All ICMJE Conflict of Interest Forms for authors and Clinical Orthopaedics and Related Research ${ }^{\circledR}$ editors and board members are on file with the publication and can be viewed on request.

Clinical Orthopaedics and Related Research ${ }^{\mathbb{R}}$ neither advocates nor endorses the use of any treatment, drug, or device. Readers are encouraged to always seek additional information, including FDAapproval status, of any drug or device prior to clinical use.

This work was performed at Mayo Clinic Arizona, Phoenix, AZ, USA.

Electronic supplementary material The online version of this article (doi:10.1007/s11999-014-4094-8) contains supplementary material, which is available to authorized users.

\section{J. F. Fraser ( $\square)$}

Department of Orthopaedic Surgery, Banner Good Samaritan Hospital, 1320 N 10th Street, Suite A, Phoenix, AZ 85006, USA e-mail: jfraser7@gmail.com
Questions/purposes The purpose of this study was to compare particle contamination at the gown-glove interface in several modern surgical helmet systems and conventional surgical gowns.

Methods A 5- $\mu$ m fluorescent powder was evenly applied to both hands to the level of the wrist flexion crease. After gowning in the standard fashion, the acting surgeon performed a 20-minute simulated TJA protocol. Each of the five gowning systems was run through five trials. The amount of gown contamination at the gown-glove interface then was measured by three observers under ultraviolet light using a grading scale from 0 (no contamination) to 4 (gross contamination). Statistical analysis was carried out with Minitab 15. Friedman's test was used to compare the levels of contamination across trials for each gown and the Mann-Whitney test was used post hoc to perform a pairwise comparison of each gown.

Results All gown-glove interfaces showed some contamination. Friedman's test showed that there was a significant difference in contamination between gowns $(\mathrm{p}=0.029)$. The Stryker T5 Zipper Toga system showed more contamination than the other gowns. The median contamination score and range for each gowning setup was 1.8 (range, 1-4; conventional Kimberly-Clark MicroCool gown without helmet), 4 (range, 3-4; Stryker T5 Zipper Toga), 3.6 (range, 0-4; Stryker helmet with conventional gown), 1.6 (range, 0-2; Stryker Flyte Toga), and 3.0 (range, 2-3; DePuy Toga). A Mann-Whitney test found no difference among any of the gowns except for the Stryker T5 Zipper Toga, which showed more contamination

S. W. Young, K. A. Valentine, N. E. Probst, M. J. Spangehl Department of Orthopaedic Surgery, Mayo Clinic Arizona, Phoenix, AZ, USA 
compared directly with each of the other four gowns ( $p<0.001$ for each gown-to-gown comparison).

Conclusions Particle contamination occurs at the gownglove interface in most commonly used positive-pressure surgical helmet systems. The Stryker T5 Zipper Toga exhibited more contamination than each of the other gowning systems.

Clinical Relevance The gown-glove interface is prone to particle contamination and all surgeons should be aware of this area as a potential source of surgical site infection. Although future studies are needed to clarify the link between particle contamination through this route and clinical infection, surgeons should consider using gowning systems that minimize the migration of fomites through the gown-glove interface.

\section{Introduction}

Infection rates as high as $9.5 \%$ were reported in some early series of total joint arthroplasty (TJA) [4]. Microbial-laden particles shed from the operating room staff were considered to be a major source of these early postoperative infections $[13,17]$. This led to the introduction of ultraclean air operating rooms and body exhaust suits. In 1983, Blomgren et al. showed a 33\% reduction in the rate of intraoperative wound culture positivity with the use of body exhaust suits compared with conventional gowns [1]. A landmark randomized clinical trial, involving more than 8000 patients who underwent TJA, demonstrated a three- to 20 -fold decrease in airborne bacterial contamination and a reduction in deep infection rates from $1 \%$ to $0.1 \%$ with the use of body exhaust suits compared with conventional attire [10]. These studies resulted in the widespread adoption of body exhaust suits by surgeons performing TJA.

The early body exhaust suits used both inflow and outflow tubing to create negative pressure within the gown and helmet system, ensuring shed particles would be drawn away from the operative site [18]. Many surgeons found this setup cumbersome, and most modern surgical helmet systems have an intake fan on the helmet itself, which draws air in through the top of the hood and, by design, out the bottom of the gown. The lack of outflow tubing creates positive pressure inside the gown. The positive pressure allows for particles shed by the operating room staff to be expelled through any hole or gap in the gown system.

A 2010 joint registry study from New Zealand showed increased infection rates with use of these modern surgical helmet systems compared with conventional gowns [7]. Other recent studies have failed to show any benefit with regard to contamination or infection rates with the use of surgical helmet systems versus conventional gowns [2, 1416]. In 2013, Young et al. investigated a single surgical helmet system and found increased particle contamination at the gown-glove interface compared with a conventional gown during simulated TJA. The researchers hypothesized that the increased infection rates observed with modern surgical helmet system use may be attributable to the egress of contaminated air at the unsealed gown-glove interface [18].

However, surgical helmet systems differ in gown material, helmet type, and one-piece toga or two-piece hood and gown systems. Some gowns have a more clothlike texture that is easily compressed at the wrist, whereas others are made from a waterproof material that is stiffer and tends to form wrinkles with deeper ravines at the gown-glove interface. These stiffer gowns may create a less airtight seal and be more prone to the migration of contaminants through the gown-glove interface. A twopiece hood and gown system may potentiate the egress of air at the hood-gown interface, thus reducing the amount of positive pressure within the gown and decreasing the egress of contaminated air at the gown-glove interface. The egress of potentially contaminated air at the gown-glove interface has not been tested in multiple surgical helmet system types.

We designed an experiment to test the hypothesis that all positive pressure, modern surgical helmet systems would demonstrate increased particle contamination when compared with conventional gowns and also to identify which, if any, of the modern surgical helmet systems are more prone to particle contamination at the gown-glove interface.

\section{Materials and Methods}

A comparative study was conducted using a standardized protocol to simulate a TJA based on a method previously described [18]. As a surrogate for bacterial-laden skin particles, which typically range in size from 5 to $15 \mu \mathrm{m}$ [5], a 5- $\mu \mathrm{m}$ fluorescent powder (Glo Germ; Hygienic Solutions, Lincoln, UK) was evenly applied to both hands of the acting surgeon (JFF or NEP) to the level of the wrist flexion crease (Fig. 1). The acting surgeon was then gowned and double-gloved (Protexis Hydrogel; Cardinal Health, Dublin, OH, USA) in the standard fashion, taking care not to contaminate the proximal portion of the glove with powder. The cuff of the gown was positioned in a standardized fashion at the level of the metacarpal phalangeal joints in an attempt to provide a longer and better seal with the glove. The gown-glove interface was then examined with an ultraviolet light to ensure no contamination had occurred during the gowning process. If contamination was detected at this point, the surgical attire was removed and gowning was performed again until no 


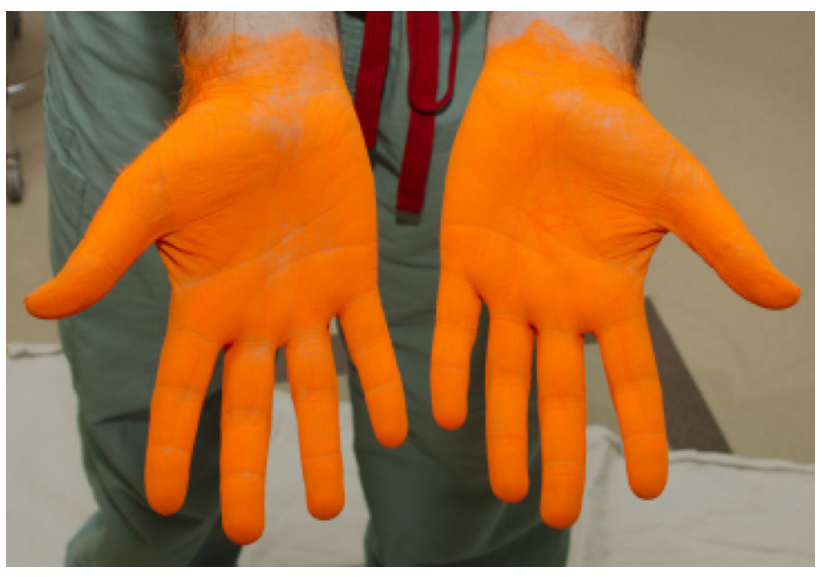

Fig. 1 The acting surgeon's hands were coated with $5 \mu \mathrm{m}$ fluorescent powder before gowning.

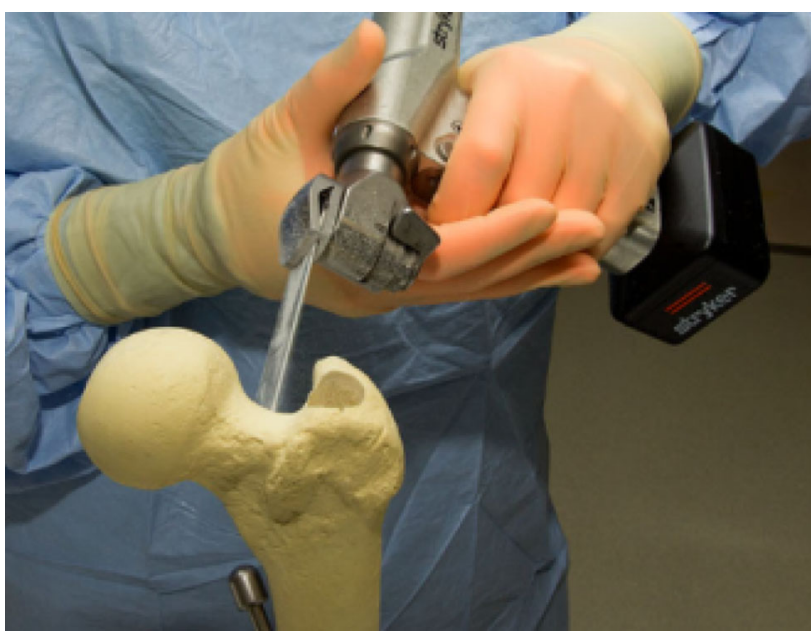

Fig. 2 A simulated TJA was performed using sawbones (Synbone, Malans, Switzerland).

preprocedure contamination was detected. A 20-minute standardized simulated TJA procedure then was performed (Fig. 2).

Simulated procedures were performed in modern operative suites without laminar flow (Appendix 1 [Supplemental materials are available with the online version of $\mathrm{CORR}^{\circledR}{ }^{\circledR}$.]). Four gown/helmet systems plus a conventional gown without a helmet were each tested five times. The high fan setting was used for each modern surgical helmet system tested (the conventional gown did not have a helmet or fan). Tested gowns included a conventional Kimberly-Clark MicroCool gown without a helmet (Irving, TX, USA), a one-piece Stryker T5 Zipper Toga with Stryker helmet (Kalamazoo, MI, USA), a MicroCool gown with a Stryker hood and helmet, a Stryker Flyte Toga with Stryker helmet, and a onepiece DePuy Toga with a DePuy helmet (Warsaw, IN, USA) (Fig. 3A-J). Contamination was graded by three observers
(KAV, MJS, SWY) under ultraviolet light using a previously published grading scale [18]: 0 (no contamination), 1 (one to five specks), 2 (five to 10 specks), 3 (10-100 specks), and 4 (> 100 specks) (Fig. 4). Each observer graded each observation independently, though observers were not blinded to other's observations. The level of contamination was further classified into four specific locations, including right and left volar and dorsal wrists.

A power calculation based on a previous study [18] suggested that five trials of each gown would be sufficient to detect true differences in degree of gown contamination between surgical helmet systems and conventional gowns. Statistical analysis was carried out with Minitab 15 (State College, PA, USA). Friedman's test was used to compare the levels of contamination across trials for each gown using the highest contamination grade of any location for each trial. The Mann-Whitney test was used post hoc to perform a pairwise comparison of each gown based on degree of contamination.

\section{Results}

The Stryker T5 Zipper Toga system showed more contamination than the other gowns. Friedman's test showed that there was a significant difference in contamination between gowns $(\mathrm{p}=0.029)$. All of the contamination grades for each observer, trial, and gown type are depicted in the dot plot (Fig. 5). Based on the Friedman test, the median contamination score and range for each gowning setup was 1.8 (range, 1-4; conventional Kimberly-Clark MicroCool gown without helmet), 4 (range, 3-4; Stryker T5 Zipper Toga), 3.6 (range, 0-4; Stryker helmet with conventional gown), 1.6 (range, 0-2; Stryker Flyte Toga), and 3.0 (range, 2-3; DePuy Toga). A MannWhitney test found no difference among any of the gowns except for the Stryker T5 Zipper Toga, which showed more contamination compared directly with each of the other four gowns $(\mathrm{p}<0.001$ for each head-to-head comparison) (Table 1).

\section{Discussion}

Infection after TJA is a devastating complication. Wound contamination at the time of the procedure is thought to be a cause of early postoperative infections $[10,13,17,18]$. Recent studies have shown increased wound contamination and infection rates with the use of modern surgical helmet systems compared with conventional gowns, and it has been hypothesized that the positive pressure within a surgical helmet system may potentially underlie the higher rates of wound contamination and infection $[7,11,12,18]$. 

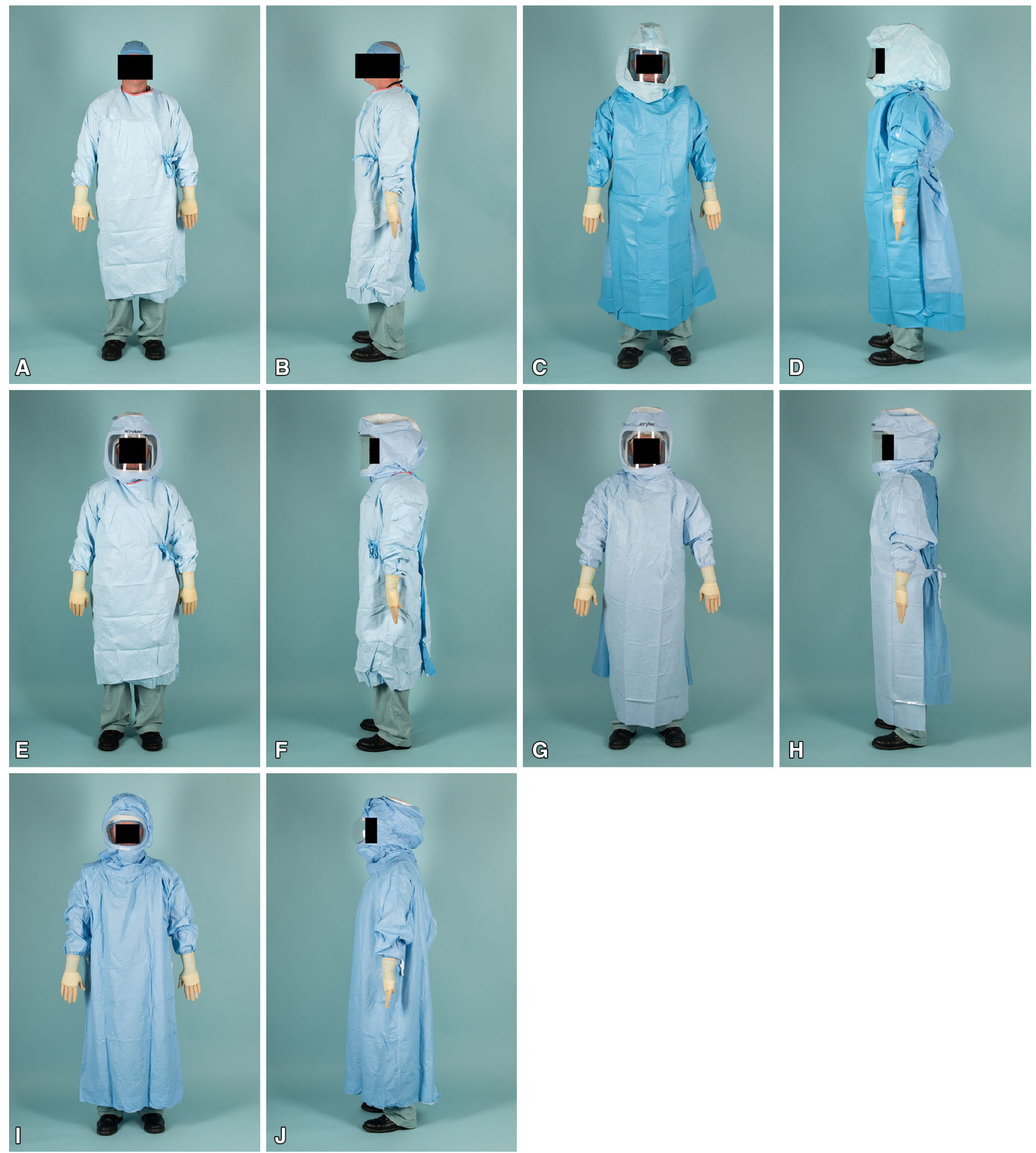

Fig. 3A-J Front and side views of each tested gowning setup included a conventional Kimberly-Clark MicroCool gown without a helmet (A-B), a one-piece Stryker T5 Zipper Toga with a Stryker

helmet $(\mathbf{C}-\mathbf{D})$, a MicroCool gown with a Stryker hood and helmet $(\mathbf{E}-$ F), a Stryker Flyte Toga with a Stryker helmet $(\mathbf{G}-\mathbf{H})$, and a onepiece DePuy Toga with a DePuy helmet (I-J).

Our study had a number of limitations. Perhaps most The purpose of our study was to determine the amount of particle contamination at the gown-glove interface in several modern surgical helmet systems as well as conventional gowns. importantly, the link between particle contamination and the clinical outcome of interest (wound infection) is unknown. Although it seems logical that a more heavily 


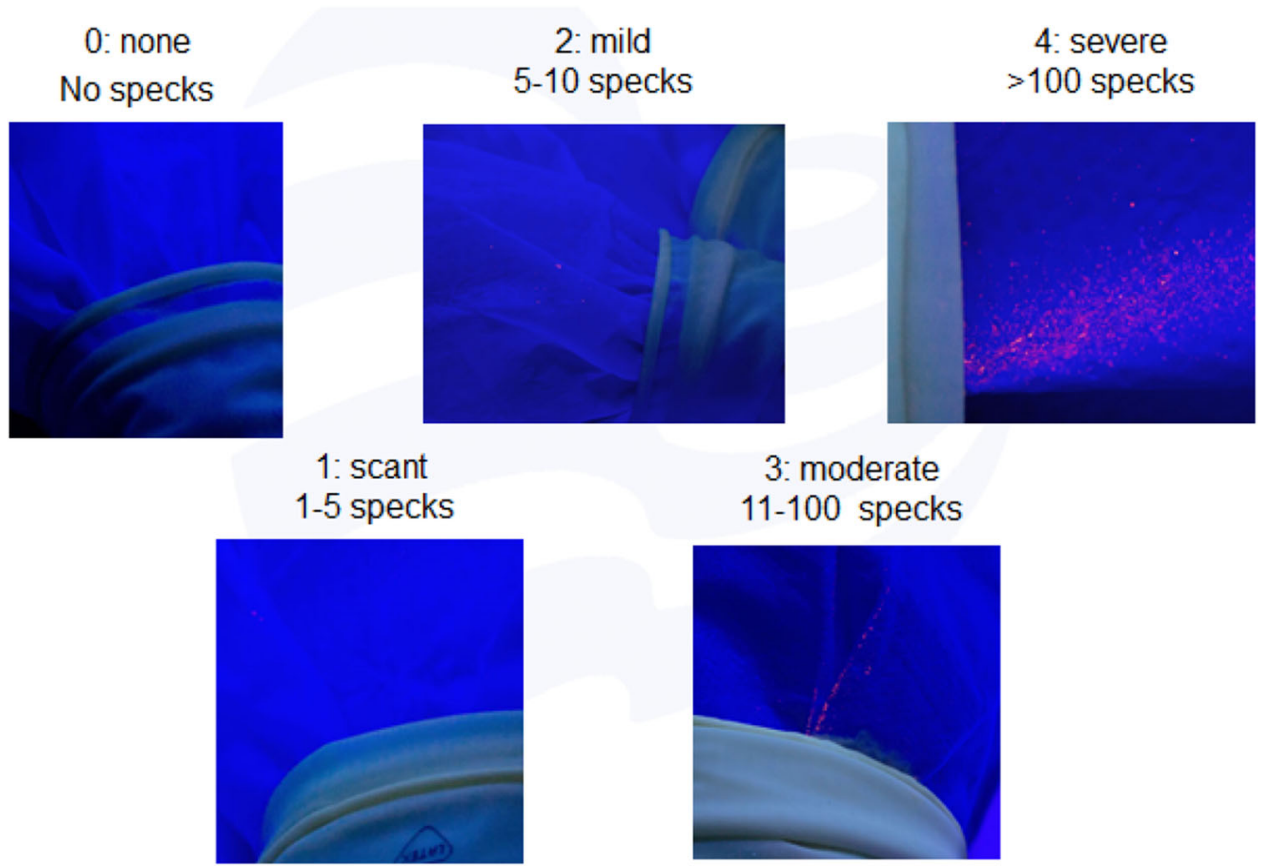

Fig. 4 To determine the degree of contamination, grading was performed by three observers (KAV, MJS, SWY) under ultraviolet light using a standardized grading scale: 0 (no contamination), 1 (one to five specks), 2 (five to 10 specks), 3 (10-100 specks), and 4 (> 100 specks).

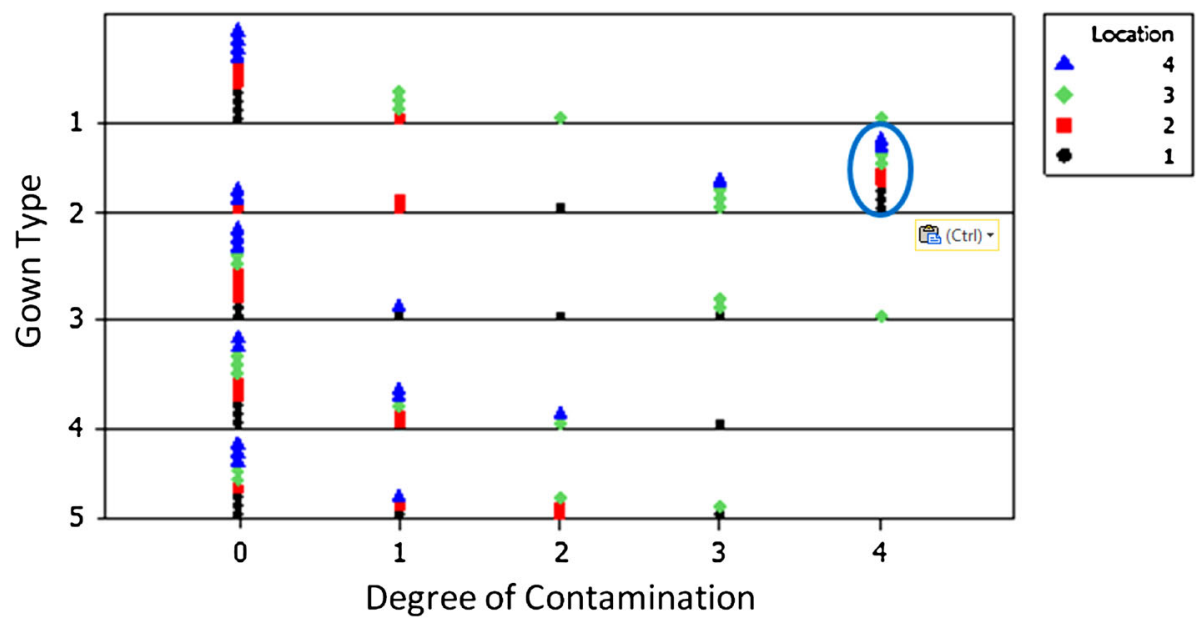

Fig. 5 A dot plot depicting gown type (y axis) versus degree of contamination (x axis) with consideration for location of contamination (black/ red: right volar/dorsal, green/blue: left volar/dorsal). Gown 2 (blue oval) showed more contamination than the other gowns.

contaminated operative site increases the risk of clinical infection, this link has been hard to prove because of the relatively low rate of infection after modern arthroplasty [3]. One recent study showed that despite having positive intraoperative wound cultures in 41 of $90(46 \%)$ patients who underwent TJA, only two patients were later diagnosed with an infection [8]. However, other studies have associated intraoperative contamination with subsequent deep infection. Knobben took four intraoperative cultures from instruments and two portions of removed bone during
100 THAs [9]. Bacterial contamination was identified in $36 \%$ of procedures, and six of $36(17 \%)$ patients with intraoperative contamination developed deep infection compared with one of $64(1.5 \%)$ patients without contamination $(p=0.008)$. This finding is supported by animal studies, and a dose-response relationship between contamination and subsequent deep infection appears to exist. Craig et al. used a rabbit TKA model to investigate the effect of contamination on subsequent deep infection and found the incidence of infection increased with the size 
Table 1. Mann-Whitney test: gown-to-gown comparison based on degree of contamination

\begin{tabular}{llllll}
\hline Gown* & 1 & 2 & 3 & 4 & 5 \\
\hline 1 & & $\mathrm{p}<0.001$ & $\mathrm{p}=0.39$ & $\mathrm{p}=0.35$ & $\mathrm{p}=0.07$ \\
2 & & & $\mathrm{p}<0.001$ & $\mathrm{p}<0.001$ & $\mathrm{p}<0.001$ \\
3 & & & & $\mathrm{p}=0.84$ & $\mathrm{p}=0.60$ \\
4 & & & & & $\mathrm{p}=0.30$ \\
5 & & & & & \\
\hline
\end{tabular}

* Tested gowns included a conventional Kimberly-Clark MicroCool gown alone (1), a Stryker T5 Zipper Toga (2), a Kimberly-Clark gown and Stryker helmet (3), a Stryker Flyte Toga (4), and a DePuy Toga (5). Gown 2 showed significantly increased contamination when compared directly with each of the other four gown systems.

of the bacterial inoculum given [6]. Such studies support a causal relationship between contamination and subsequent deep infection; therefore, it is reasonable to identify and attempt to minimize potential sources of contamination such as the gown-glove interface. It remains difficult to quantify the relative importance of such sources to clinical deep infection rates and this is an area for future study.

We based our trial number on a power analysis designed to detect a difference in contamination between a modern surgical helmet system and a conventional gown [18]. We chose a 20-minute simulated procedure because a previous study demonstrated that a similar length protocol was long enough to detect significant amounts of gown contamination [18]. Despite our small sample size, our study evaluated more gown types and performed more trials per gown than any previous study. The fluorescent powder was chosen as a surrogate for skin squames based on its size and previous use in the literature [5, 18], but the use of this powder as a surrogate is limited based on unknown differences between its ability to migrate to the outside of a gown compared with real-life fomites shed from operative personnel.

Finally, blinding the surgeons performing the simulated procedures or the observers to the gown type was not possible, because each gown had obvious characteristics and styles that were unique. Although photographs and remote observations may have permitted some degree of blinding, these strategies would have been challenged by the folds of each gown that were present at the gown-glove interface and often blocked areas of contamination from direct visualization. Direct visualization of each gownglove interface allowed for a more thorough investigation to detect any areas of notable contamination. Thus, not blinding may have actually strengthened our study and improved our sensitivity to detect particulate contamination compared with feasible blinding options.

The Stryker T5 Zipper Toga produced the greatest degree of contamination at the glove-gown interface. Our results do not support the positive pressure hypothesis as the main driver of particulate contamination at the gown-glove interface, because we did not see increased contamination in any of the other ventilated gowns compared with the conventional gown. Although we did not specifically control or test for gown stiffness as a variable, we did observe that the gowns with a stiffer, more waterproof material seemed to create deeper folds and a less airtight seal at the gown-glove interface. In addition, the stiffer gown material tended to increase the distal migration of the proximal glove edge, essentially displacing the gown-glove interface closer to the source of contamination (ie, the hands). Future studies should control both for gown stiffness and distal glove migration.

The link between staff-sourced contamination and clinical infection rates in TJA must also be further tested, perhaps through studies that genetically match organisms that have caused a clinical infection to microbes found on operative personnel. It is a reasonable goal for all surgeons to make every effort to minimize the amount of staffsourced wound contamination. The findings of our study suggest that some degree of contamination at the cuffgown interface occurs with most commonly used gowning systems. The junction of the gown and glove in modern surgical helmet systems is not sterile and in fact should be considered an area of particular vulnerability in terms of contamination. A better gown-glove interface is needed to reduce particle contamination at this interface.

Acknowledgments We thank Nathan Pallace for his assistance with the photography during this project, and Ryan McLemore $\mathrm{PhD}$, for his help with the statistical analysis.

\section{References}

1. Blomgren G, Hambraeus A, Malmborg AS. The influence of the total body exhaust suit on air and wound contamination in elective hip-operations. J Hosp Infect. 1983;4:257-268.

2. Bohn WW, McKinsey DS, Dykstra M, Koppe S. The effect of a portable HEPA-filtered body exhaust system on airborne microbial contamination in a conventional operating room. Infect Control Hosp Epidemiol. 1996;17:419-422.

3. Bozic KJ, Grosso LM, Lin Z, Parzynski CS, Suter LG, Krumholz HM, Lieberman JR, Berry DJ, Bucholz R, Han L, Rapp MT, Bernheim S, Drye EE. Variation in hospital-level risk-standardized complication rates following elective primary total hip and knee arthroplasty. J Bone Joint Surg Am. 2014;96:640-647.

4. Charnley J. A clean-air operating enclosure. $B r \quad J$ Surg. 1964;51:202-205.

5. Clark RP, Reed PJ, Seal DV, Stephenson ML. Ventilation conditions and air-borne bacteria and particles in operating theatres: proposed safe economies. J Hyg (Lond). 1985;95:325-335.

6. Craig MR, Poelstra KA, Sherrell JC, Kwon MS, Belzile EL, Brown TE. A novel total knee arthroplasty infection model in rabbits. J Orthop Res. 2005;23:1100-1104.

7. Hooper GJ, Rothwell AG, Frampton C, Wyatt MC. Does the use of laminar flow and space suits reduce early deep infection after total hip and knee replacement? The ten-year results of the New Zealand Joint Registry. J Bone Joint Surg Br. 2011;93:85-90. 
8. Jonsson EÖ, Johannesdottir H, Robertsson O, Mogensen B. Bacterial contamination of the wound during primary total hip and knee replacement. Median 13 years of follow-up of 90 replacements. Acta Orthop. 2014;85:159-164.

9. Knobben BAS, Engelsma Y, Neut D, van der Mei HC, Busscher HJ, van Horn JR. Intraoperative contamination influences wound discharge and periprosthetic infection. Clin Orthop Relat Res. 2006;452:236-241.

10. Lidwell OM, Lowbury EJ, Whyte W, Blowers R, Stanley SJ, Lowe D. Effect of ultraclean air in operating rooms on deep sepsis in the joint after total hip or knee replacement: a randomised study. Br Med J (Clin Res Ed). 1982;285:10-14.

11. Namba RS, Inacio MCS, Paxton EW. Risk factors associated with surgical site infection in 30,491 primary total hip replacements. $J$ Bone Joint Surg Br. 2012;94:1330-1338.

12. Namba RS, Inacio MCS, Paxton EW. Risk factors associated with deep surgical site infections after primary total knee arthroplasty: an analysis of 56,216 knees. J Bone Joint Surg Am. 2013;95:775-782.
13. Owers KL, James E, Bannister GC. Source of bacterial shedding in laminar flow theatres. J Hosp Infect. 2004;58:230-232.

14. Pasquarella C, Pitzurra O, Herren T, Poletti L, Savino A. Lack of influence of body exhaust gowns on aerobic bacterial surface counts in a mixed-ventilation operating theatre. A study of 62 hip arthroplasties. J Hosp Infect. 2003;54:2-9.

15. Shaw JA, Bordner MA, Hamory BH. Efficacy of the Steri-Shield filtered exhaust helmet in limiting bacterial counts in the operating room during total joint arthroplasty. $J$ Arthroplasty. 1996;11:469-473.

16. Der Tavitian J, Ong SM, Taub NA, Taylor GJS. Body-exhaust suit versus occlusive clothing. A randomised, prospective trial using air and wound bacterial counts. J Bone Joint Surg Br. 2003;85:490-494.

17. Whyte W, Hodgson R, Tinkler J. The importance of airborne bacterial contamination of wounds. J Hosp Infect. 1982;3:123-135.

18. Young SW, Chisholm C, Zhu M. Intraoperative contamination and space suits: a potential mechanism. Eur J Orthop Surg Traumatol Orthop Traumatol. 2014;24:409-413. 